\title{
Galectin-1 expression in activated pancreatic satellite cells promotes fibrosis in chronic pancreatitis/pancreatic cancer via the TGF- $\beta 1 /$ Smad pathway
}

\author{
DONG TANG $^{1 *}$, QI WU ${ }^{4 *}$, JINGQIU ZHANG $^{1 *}$, HONGPENG ZHANG $^{1}$, ZHONGXU YUAN $^{2}$, \\ JIAMING XU ${ }^{1}$, YANG CHONG ${ }^{1}$, YUQIN HUANG ${ }^{1}$, QINGQUAN XIONG ${ }^{1}$, SEN WANG ${ }^{3}$, \\ YING TIAN $^{4}$, YONGDIE LU $^{4}, \mathrm{XIAO} \mathrm{GE}^{4}$, WENJING SHEN ${ }^{4}$ and DAORONG WANG ${ }^{1}$
}

\begin{abstract}
${ }^{1}$ Department of General Surgery, Institute of General Surgery, Northern Jiangsu Provincial People's Hospital, Clinical Medical College, Yangzhou University, Yangzhou, Jiangsu 225001; ${ }^{2}$ Department of General Surgery, Anhui Second Provincial People's Hospital, Hefei, Anhui 230000; ${ }^{3}$ Department of General Surgery, The First Affiliated Hospital of Nanjing Medical University, Nanjing, Jiangsu 210029; ${ }^{4}$ Department of Clinical Medicine, Medical College of Yangzhou University, Yangzhou, Jiangsu 225001, P.R. China
\end{abstract}

Received July 24, 2017; Accepted December 12, 2017

DOI: $10.3892 / 0 r .2018 .6202$

\begin{abstract}
Chronic pancreatitis/pancreatic cancer (CP/PC) is characterized by fibrous connective tissue proliferation induced by activated pancreatic stellate cells (PSCs). Galectin-1 is upregulated in activated PSCs and is important for the continuing activation of PSCs. The aim of this study was to evaluate the effect of galectin-1 derived from activated PSCs on the progression of fibrosis in $\mathrm{CP} / \mathrm{PC}$. To this end, the expression of desmin, $\alpha$-SMA, galectin-1, fibronectin and collagen type I in normal pancreatic, CP and PC tissues, as well as quiescent/ activated PSCs, was investigated. The proliferation rate and migration ability of control, galectin-1-overexpressing and galectin-1-silenced PSCs were also evaluated, as well as the mRNA and protein expression of fibronectin, collagen type I, $\alpha$-SMA, tissue inhibitors of metalloproteinases (TIMP)-1, MMP-2, Smad 2 and TGF- $\beta 1$. Furthermore, the effect of adding a TGF- $\beta 1$ receptor inhibitor on the expression of these proteins was examined. The results revealed that the expression profile of desmin, $\alpha$-SMA, galectin-1, fibronectin and collagen type I in the normal pancreas was similar to that of quiescent PSCs and the expression profile in $\mathrm{CP} / \mathrm{PC}$ tissues was similar to that of
\end{abstract}

Correspondence to: Dr Dong Tang or Professor Daorong Wang, Department of General Surgery, Institute of General Surgery, Northern Jiangsu Province People's Hospital, Clinical Medical College, Yangzhou University, 98 West Nantong Road, Yangzhou, Jiangsu 225001, P.R. China

E-mail: 83392785@qq.com

E-mail: daorong666@sina.com

*Contributed equally

Key words: chronic pancreatitis, pancreatic cancer, pancreatic stellate cell, galectin-1, fibrosis activated PSCs. Furthermore, galectin-1-overexpressing PSCs exhibited a significantly higher proliferation rate and migration ability, while galectin-1-silenced PSCs exhibited a significantly lower proliferation rate and migration ability than the control PSCs. The expression of fibronectin, collagen type I, $\alpha$-SMA, MMP-2 and TIMP-1 was also significantly higher in the galectin-1-overexpressing PSCs than the control PSCs and this effect was found to be mediated by the TGF- $\beta 1 /$ Smad pathway. The trends in the expression of these factors were reversed in the galectin-1-silenced PSCs. From these findings, it can be concluded that overexpression of galectin-1 promotes PSC activity (proliferation and migration) and stimulates fibrosis by increasing extracellular matrix synthesis and decreasing the MMP/TIMP ratio via the TGF- $\beta 1 /$ Smad pathway. Thus, galectin-1 may be a novel candidate for reversing or halting fibrosis progression in $\mathrm{CP} / \mathrm{PC}$.

\section{Introduction}

Chronic pancreatitis (CP) is a serious disease that is characterized by progressive inflammation of the pancreas and fibrosis, which result in exocrine and endocrine dysfunction (1). Pancreatic fibrosis is closely associated with $\mathrm{CP}$ and pancreatic cancer (PC) and it induces severe damage in the pancreas. CP and PC are characterized by a desmoplastic reaction that involves activated pancreatic stellate cells (PSCs) (1-3). This reaction promotes the growth and invasion of tumor cells (3-5). The activation of PSCs has been previously proposed as the key initiating step in pancreatic fibrosis (6) and a major source of extracellular matrix (ECM) deposition during pancreatic injury (7). Activated PSCs are believed to significantly contribute to the progression of pancreatic diseases and may therefore, present beneficial therapeutic targets $(3,8-11)$. Furthermore, the activation of PSCs is associated with the secretion of various inflammatory cytokines/chemokines, as well as collagen $(12,13)$. Understanding the mechanism underlying the activation of 
PSCs and the effects of activated PSCs on pancreatic diseases would help identify treatment targets for pancreatic fibrosis associated with diseases such as $\mathrm{CP} / \mathrm{PC}$.

Transforming growth factor (TGF)- $\beta$, a potent pro-fibrotic factor that plays a functional role in the pathogenesis of pancreatic fibrosis (14), is responsible for the activation of PSCs (15). Following stimulation with TGF- $\beta$, PSCs exhibit enhanced expression of significant ECM proteins, including collagen and fibronectin. Concurrently, TGF $\beta$ inhibits the degradation of ECM by blocking the secretion of proteases, such as matrix metalloproteinases (MMPs) and stimulating the production of naturally occurring protease inhibitors, such as tissue inhibitors of metalloproteinases (TIMPs) (16). To date, previous studies have revealed that PSCs are the main source of ECM proteins in pancreatic fibrosis (16) and that the activation and collagen synthesis of PSCs are highly controlled by TGF- $\beta 1$ (17). TGF- $\beta 1$ is a subtype of the TGF- $\beta$ family, which is multifunctional and increases significantly in $\mathrm{CP}$ (in both human and animal models) (18).

Galectins are a growing family of $\beta$-galactoside-binding animal lectins that have been implicated in a variety of biological processes, including fibrosis, angiogenesis and immune activation (19). Galectin-1, a member of the galectin family, is strongly expressed in fibroblasts, which have been recognized as activated PSCs, in CP/PC (20). Galectin-1 has high affinity for $\beta$-galactosides and induces collagen synthesis, chemokine production and proliferation of PSCs in CP/PC $(7,20,21)$. In addition, previous studies have revealed that galectin-1 plays a role in the desmoplastic reaction associated with PC (22). Furthermore, it has been reported that TGF- $\beta 1$ alone or TGF- $\beta 1$ together with galectin- 1 induces the transition of human dermal fibroblasts to myofibroblasts (23) and that galectin-1 may promote the TGF- $\beta 1$-induced differentiation of fibroblasts by sustaining nuclear localization of Smad2 in pulmonary fibrotic diseases (17). However, it is not known whether galectin-1 plays a fibrogenic role in $\mathrm{CP} / \mathrm{PC}$ and which is the related underlying mechanism. Therefore, the present study was designed to investigate the potential fibrogenic role of galectin-1 in activated PSCs in CP/PC using immunohistochemical methods under in vitro conditions.

\section{Materials and methods}

Patients and pancreatic tissues. The clinicopathological characteristics of patients, the PDAC, CP and normal pancreatic control tissues, as well as the histological evaluation of these specimens have been previously described $(21,24,25)$. From January 2006 to December 2010, PC tissue samples were obtained from 66 patients undergoing pancreaticoduodenectomy for PC and from 18 patients with $\mathrm{CP}$ at the First Affiliated Hospital of Nanjing Medical University, (Jiangsu, China). The PC patients comprised 45 men and 21 women with a median age of 55 years (range, 37-83 years) and the CP patients comprised 13 men and five women with a median age of 54.5 years (range, 27-71 years). Ten normal pancreatic control tissue samples were obtained from patients undergoing partial pancreatic resections for bile duct or duodenal ampullary cancer. All procedures performed involving human participants were in accordance with the ethical standards of the ethics committee of The First Affiliated Hospital of Nanjing Medical University and with the 1964 Helsinki declaration and its later amendments or comparable ethical standards.

Compliance with ethical standards. Informed consents were obtained from all the patients for their participation in the study, which was approved by the ethics committee of The First Affiliated Hospital of Nanjing Medical University (Jiangsu Provincial People's Hospital). Every participant provided a written informed consent to participate in this study and the copies of the written consents of participants were reserved in our laboratory and can be obtained at any time. The participants signed the Letter of Information and Consent and each one held and saved a copy of the informed consent. The ethics committee approved this consent procedure, which has been recorded in the Consent Form of the Ethics Committee.

Cell and culture conditions. Primary human PSCs were isolated, identified, maintained and passaged as previously described $(21,24,25)$. The cells from passage numbers $0-5$ were used for all assays.

Preparation and transduction of recombinant lentiviruses. The plasmids used for preparing the recombinant lentiviruses have been previously described (21). Briefly, the galectin-1 gene fragment was excised from a human cDNA library and cloned into pHAGE-CMV-MCS-IZsGreen between the BamHI and XhoI restriction sites. Galectin-1-specific oligonucleotides were ligated into the pLKO.1-puro vector (21). The study groups were as follows: overexpression Galectin-1-PSC (Over), normal PSC control group (Control), knockdown shRNA-Galectin-1-PSC\#1 (Sh-1) and shRNA-Galectin1-PSC \#2 (Sh-2).

Wound healing assay. PSCs were seeded in 24-well culture plates and grown to reach confluency. After starvation for $12 \mathrm{~h}$, the monolayers were wounded by scrapping off a strip of cells with a 200- $\mu \mathrm{l}$ pipette tip. The cells were incubated for $24 \mathrm{~h}$. Subsequently the cells were fixed, images of three different segments of the 'wound' area on each well were captured at an x10 magnification, using the Olympus DP71 camera (Olympus Optical Co. Ltd, Tokyo, Japan) and the cell numbers inside the wound boundaries were counted.

In vitro migration assay. PSC migration through Matrigel was determined using 6-well Corning Transwell chambers (8.0- $\mu \mathrm{m}$ pore size with a polycarbonate membrane) as previously described (26). Briefly, the upper chambers were coated with diluted Matrigel $(1 \mathrm{mg} / \mathrm{ml}, 356243$; BD Biosciences, Bedford, MA, USA) and incubated at $37^{\circ} \mathrm{C}$ in $5 \% \mathrm{CO}_{2}$ for $3 \mathrm{~h}$. After trypsinization, PSCs were suspended in DMEM with $10 \%$ FBS at $1 \times 10^{5}$ cells/well and immediately placed onto the upper compartment. After 24-h incubation, nonmigrated cells were removed from the upper surface of the membrane by wiping with cotton-tipped swabs. The cells on the lower surface of the membrane were stained with $0.1 \%$ crystal violet for $10 \mathrm{~min}$ and photographed at an x10 magnification using the Olympus DP71 camera (Olympus Optical). The crystal violet was then bleached using $500 \mu 133 \%$ acetic acid. Absorbance at $570 \mathrm{~nm}$ was determined using a microtiter plate reader. 
Table I. Primers used for quantitative real-time RT-PCR.

\begin{tabular}{lll}
\hline Primers & \multicolumn{1}{c}{ Forward sequence 5'-3' } & \multicolumn{1}{c}{ Reverse sequence 5'-3' } \\
\hline Galectin-1 & GAGGTGGCTCCTGACGCTAA & CCTTGCTGTTGCACACGATG \\
TGF- $\beta 1$ & GAAACCCACAACGAAATCTATGAC & GCTGAGGTATCGCCAGGAAT \\
Smad2 & TCTTGATGGTCGTCTCCAGGTA & AGAGGCGGAAGTTCTGTTAGG \\
MMP-2 & CCTTTGCTCGTGCCTTCCA & TCGGCGTTCCCATACTTCA \\
TIMP-1 & GGCTTCTGGCATCCTGTTGT & GTGGTCTGGTTGACTTCTGGTG \\
$\alpha-$ SMA & GGTGACGAAGCACAGAGCAA & ACCGCCTGGATAGCCACATAC \\
Collagen type I & GCATTCGTGGCGATAAGGG & ACCAGCGATACCAGGCAGA \\
Fibronectin & CGACTGTGGACCAAGTTGATGAC & AAGGTTGAGTTCTGTGCTGCTAC \\
$\beta$-actin & AGAAAATCTGGCACCACACC & TAGCACAGCCTGGATAGCAA \\
\hline
\end{tabular}

In vitro proliferation assay. PSC proliferation was determined by methyl thiazolyl tetrazolium (MTT) assay (Sigma-Aldrich, St. Louis, MO, USA) as previously described (27). PSCs $\left(5 \times 10^{5}\right)$ were seeded in 6-well plates, cultured with $10 \%$ fetal culf serum (FCS) for $12 \mathrm{~h}$ until the cells adhered to the plate and then, exchanged the medium and the proliferation was detected at 24, 48, $72 \mathrm{~h}$. The results were expressed as absorbance at $570 \mathrm{~nm}$ in the microtiter plate reader.

Western blot analysis. Western blotting was performed as previously described $(21,24,25)$. The following antibodies were used: mouse anti-Galectin-1 antibodies (1:200, sc-166618; Santa Cruz Biotechnology, Santa Cruz, CA, USA), anti- fibronectin antibodies (1:200, sc-59824; Santa Cruz Biotechnology), anti-collagen type I antibodies (1:200, sc-376350; Santa Cruz Biotechnology), anti- $\alpha$-SMA antibodies (1:200, MA1-37027; Thermo Fisher Scientific Inc., Fremont, CA, USA), anti-TIMP-1 antibodies (1:200, sc-21734; Santa Cruz Biotechnology), antiMMP-2 antibodies (1:200, sc-13595; Santa Cruz Biotechnology) or anti-Smad2 antibodies (1:200, sc-101153; Santa Cruz Biotechnology).

Quantitative reverse transcription-polymerase chain reaction. Total RNA was extracted from all the cultured groups of PSCs using TRIzol reagent (Invitrogen Life Technologies, Beijing, China) according to the manufacturer's instructions. Quantitative reverse transcription-polymerase chain reaction (qRT-PCR) was performed as previously described $(21,24,25)$. The sequences of primers used in the present study are shown in Table I.

Immunohistochemical staining and evaluation. Pancreatic tissue samples were fixed by immersion in $4 \%$ paraformaldehyde overnight at $4{ }^{\circ} \mathrm{C}$ and then embedded in regular paraffin wax and cut into $4-\mu \mathrm{m}$ sections. Immunohistochemical detection and analyses were performed as previously described $(21,24,25)$. The primary antibodies used were as follows: mouse monoclonal anti-Galectin-1, anti-fibronectin antibodies, anti-collagen type I antibodies, anti- $\alpha$-SMA antibodies, or anti-Desmin antibodies. The results of the immunohistochemical staining were evaluated by two experienced pathologists.

Statistical analysis. Values are expressed as the mean \pm standard deviation (SD). All experiments were performed in triplicate. One way ANOVA and t-tests were performed using SPSS 13.0 software (SPSS Inc., Chicago, IL, USA) to compare differences between groups. All P-values were two-sided and $\mathrm{P}$-values $<0.05$ were considered to indicate a statistically significant difference.

\section{Results}

Role of activated PSCs in fibrosis associated with CP/PC. Desmin and $\alpha$-SMA are important markers of the quiescent and activation statuses of PSCs, respectively $(21,28)$. Activation of PSCs is regulated by a complex network of growth factors and cytokines and is associated with increase in the expression and release of collagen I and II, fibronectin and other components of the ECM in PSCs (29-31). With the development in the deposition of ECM components, pancreatic fibrosis gradually increases. To understand the role of PSCs in pancreatic fibrosis, we performed immunohistochemical staining for desmin, $\alpha$-SMA, fibronectin and collagen type I in normal pancreatic, $\mathrm{CP}$ and PC tissues. Immunohistochemical staining revealed that desmin was positively expressed and that $\alpha$-SMA, fibronectin and collagen type I were negatively or weakly expressed in the normal pancreas, in which the expression profile was consistent with that of PSCs in the quiescent stage (Fig. 1). However, desmin was weakly expressed and $\alpha$-SMA, fibronectin and collagen type I were positively expressed in $\mathrm{CP} / \mathrm{PC}$ tissues, in which the expression profile was consistent with that of PSCs in the activation stage (Fig. 1). In addition, galectin-1 was negatively expressed in normal pancreatic tissue and quiescent PSCs and was positively expressed in CP tissue and activated PSCs. Thus, galectin-1 expression was also associated with the activation stage of PSCs and the degree of fibrosis of pancreatic tissue (Fig. 1).

Effect of galectin-1 on the proliferation of PSCs. In order to obtain PSCs with different expression levels of galectin-1 that reflect different pancreatic conditions, lentiviral sh1RNAgalectin-1 and sh2RNA-galectin-1 transduction in PSCs was performed. PSCs that overexpressed galectin-1 were purified by screening for green fluorescent protein (GFP) using flow cytometry and PSCs that contained the galectin-1silenced plasmids (sh1RNA- and sh2RNA-transduced galectin-1-knockdown PSCs) were selected with puromycin. 


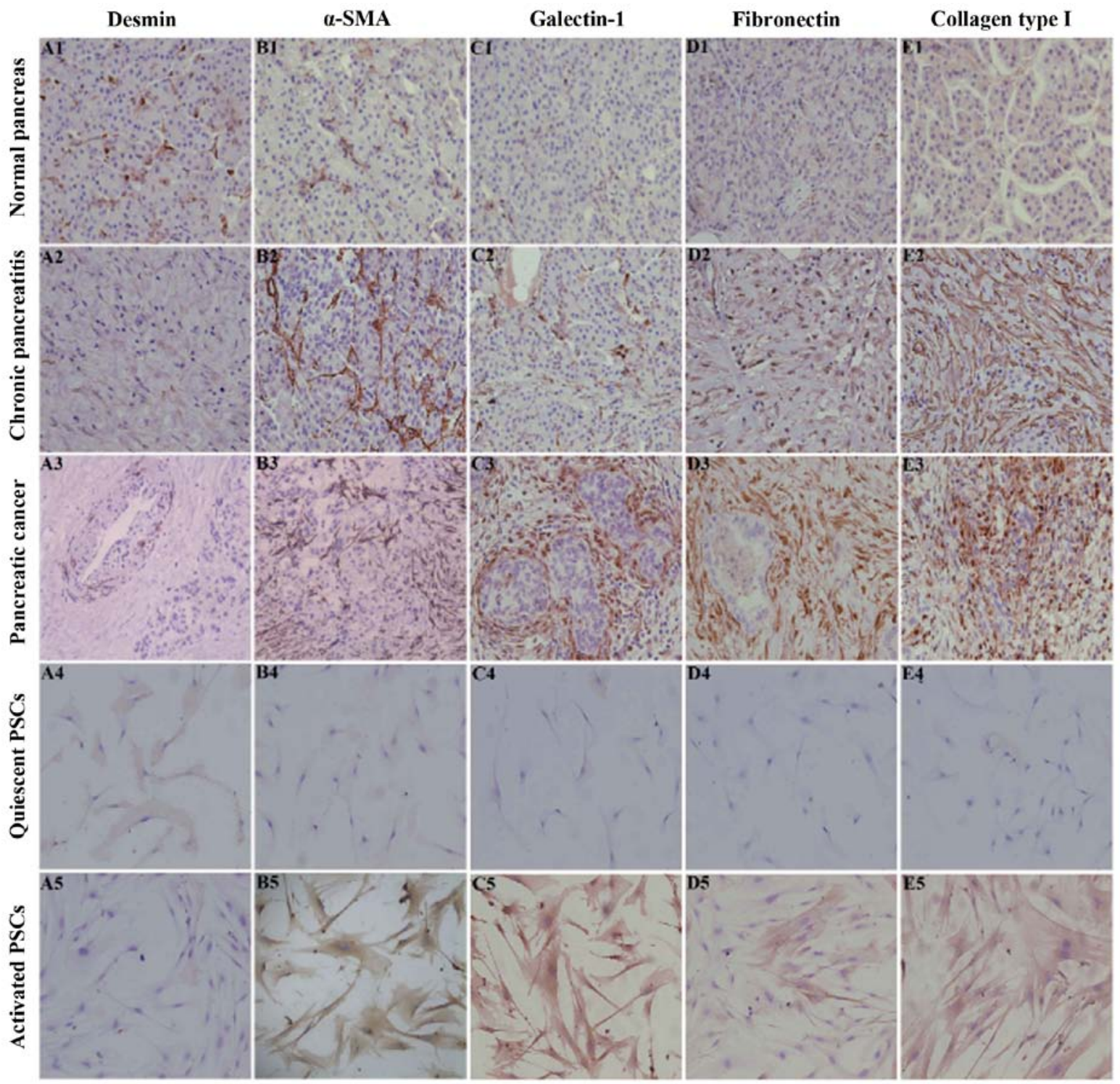

Figure 1. Immunohistochemical staining of desmin (A), $\alpha$-SMA (B), galectin-1 (C), fibronectin (D) and collagen type I (E) in normal pancreatic tissue (A1-E1), chronic pancreatic tissue (A2-E2), PC tissue (A3-E3), quiescent PSCs (A4-E4) and activated PSCs (A5-E5). Immunohistochemical staining revealed that desmin (A1) was positively expressed while $\alpha$-SMA (B1), fibronectin (C1) and collagen type I (D1) were negatively or weakly expressed in the normal pancreas, in which the expression profile was consistent with that of quiescent PSCs (A4-E4). In contrast, desmin (A2) was weakly expressed and $\alpha$-SMA (B2), fibronectin (D2) and collagen type I (E2) were positively expressed in CP and PC tissues (A3-E3), in which the expression profile was consistent with that of activated PSCs (A5-E5). Quiescent PSCs were obtained on the second day and activated PSCs were obtained on the seventh day of the primary culture. In all the images, immunohistochemical staining is indicated by the brown diaminobenzidine color reaction and the sections are counterstained with hematoxylin. Original magnification, $\mathrm{x} 200$. CP, chronic pancreatitis; PC, pancreatic cancer.

The MTT assay results revealed that galectin-1-overexpressing PSCs had a significantly higher proliferation rate than the control PSCs $(\mathrm{P}<0.05)$. Furthermore, the galectin-1-silenced PSCs had a significantly lower proliferation rate than the control PSCs $(\mathrm{P}<0.05)$ (Fig. 2C).

Effect of galectin-1 on the migration of PSCs. PSC migration was assessed by the wound healing assay, a well-established in vitro system for assessing cell motility. A confluent monolayer growing in 24-well plates was wounded by scraping off the cells with a pipette tip, thus creating a space free of cells. The cells were allowed to migrate into the cell-free area. In the presence of 5\% FCS, the migration of galectin-1-overexpressing PSCs $(\mathrm{P}<0.05)$ was significantly greater than that of the control PSCs. The migration of galectin-1-silenced PSCs (transduced with sh1RNA and sh2RNA) was significantly lower than that of the control PSCs $(\mathrm{P}<0.05)$ (Fig. 2A and $\mathrm{D})$. The migration ability of the different groups of PSCs was further confirmed by the Transwell assay. The results of this assay also revealed that the migration ability of galectin-1-overexpressing PSCs was significantly greater than that of the control PSCs, and that the migration ability of the galectin-1-silenced PSCs was significantly lower than that of the control PSCs $(\mathrm{P}<0.05$; Fig. 2B and E).

Effect of galectin-1 expression on the level of MMP-2, TIMP-1 and other fibrosis-associated factors in activated PSCs. In pancreatic fibrosis, there is an imbalance in the synthesis and degradation of the ECM. MMPs and TIMPs are mainly responsible for the degradation of the pancreatic 


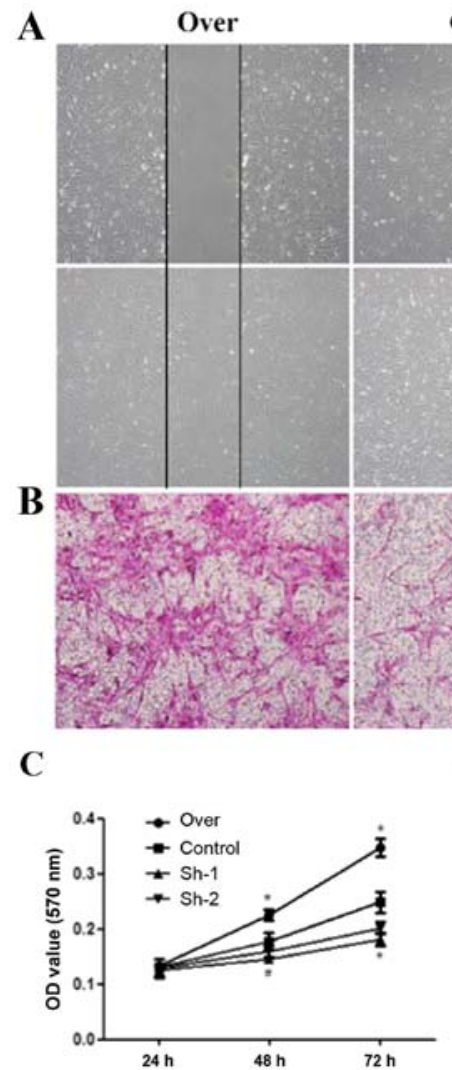

Control Sh-1 Sh-2
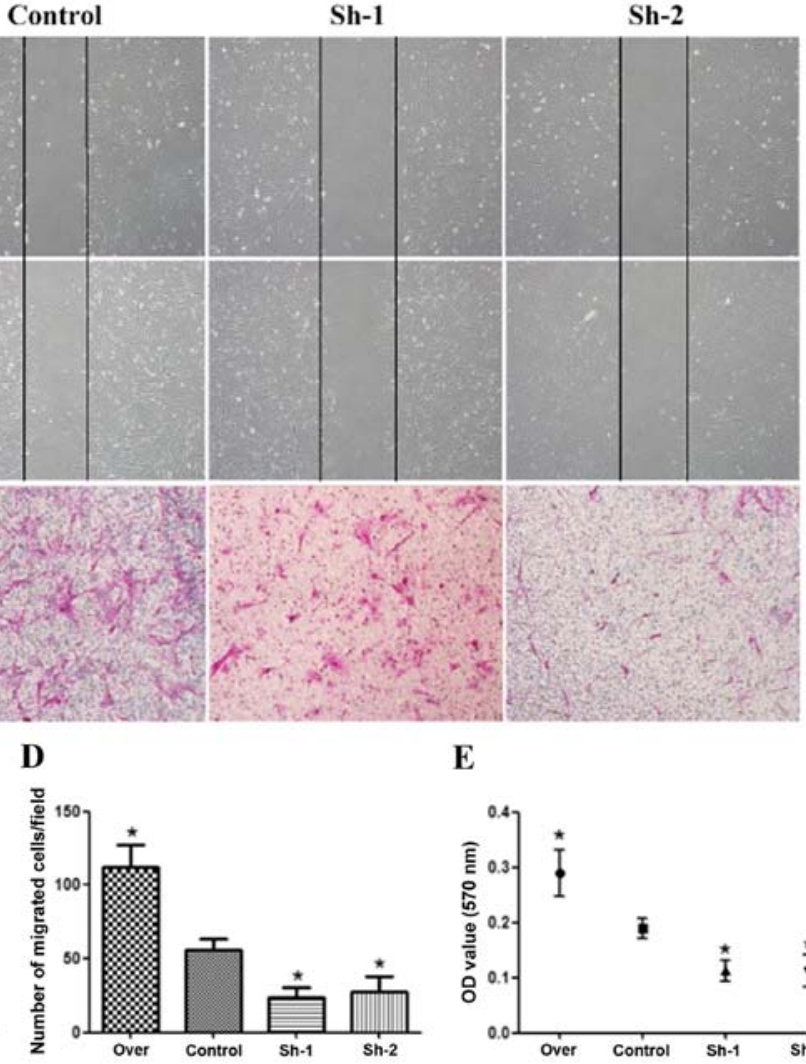

$\mathbf{E}$

Figure 2. Effect of galectin-1 on the migration and proliferation of cultured human PSCs. (A and D) Effect of differential expression of galectin-1 on PSCs was assessed by the wound healing assay, which revealed that the migration of galectin-1-overexpressing PSCs (over) was significantly greater than that of naive PSCs (control) and that the migration of galectin-1-knockdown PSCs (Sh-1 and Sh-2) was significantly lower than that of the control PSCs. "P<0.05 Over vs. Control; "P $<0.05$ Sh-1 vs. control; "P $<0.05$ Sh-2 vs. control; $\mathrm{P}>0.05$ Sh-2 vs. Sh-1. The migration ability of the different groups of PSCs was further confirmed by an in vitro Transwell migration assay (B and E). (C) The MTT assay revealed that the proliferation rate of the Over group was significantly higher than that of the Control group and that the proliferation rates of the Sh-1 and Sh-2 groups were significantly lower than that of the Control group. ${ }^{*} \mathrm{P}<0.05$ vs. control PSCs. Data are expressed as the means \pm SEM ( $n=3$ experiments/group). PSCs, pancreatic stellate cells.

ECM. MMPs play a role in the degradation of collagen, while TIMPs have an inhibitory effect on MMPs (32-34). Our quantitative PCR and western blot analysis results indicated that in galectin-1-overexpressing PSCs, the expression of both MMP-2 and TIMP-1 was increased, but the expression of TIMP-1 increased to a higher degree than the expression of MMP-2 ( $\mathrm{P}<0.01$; Fig. 3A and B). In addition, galectin-1-overexpressing PSCs also revealed an increase in the expression of fibronectin, collagen type I and $\alpha$-SMA (Fig. 3A and B), which are fibroblast markers that are strongly expressed in activated PSCs, as well as TGF- $\beta 1$ expression and Smad2 phosphorylation. In contrast, in galectin-1-silenced PSCs, the opposite effects were observed (Fig. 3A and B); lower galectin-1 expression resulted in lower MMP-2 and slightly lower TIMP-1 expression in galectin-1-silenced PSCs than in the control PSCs $(\mathrm{P}<0.05$; Fig. $3 \mathrm{~A}$ and $\mathrm{B})$, but a significant decrease was observed in the expression of fibronectin, collagen type I and $\alpha$-SMA. Collectively these results indicated that overexpression of galectin-1 promoted the fibrosis of activated PSCs by tilting the balance of MMP/TIMP expression in favor of TIMP and that galectin-1 silencing reversed this effect on the progression of fibrosis.

Endogenous galectin-1-induced fibrosis of activated PSCs via the TGF- $\beta 1 /$ Smad signaling pathway. As aforementioned, galectin-1 overexpression in PSCs resulted in an increase in both TGF- $\beta 1$ and Smad2 expression and was also associated with MMP/TIMP imbalance. It is not clear whether the TGF- $\beta 1 /$ Smad 2 signaling pathway is directly associated with MMP/TIMP imbalance and fibrosis of PSCs. Therefore, in order to shed light on the role of this pathway in pancreatic fibrosis, control, galectin-1-overexpressing and galectin-1silenced PSCs were treated for $48 \mathrm{~h}$ with SB525334, which is a selective inhibitor of TGF- $\beta$ receptor I (ALK5, TGF- $\beta$ RI). This agent inhibits TGF- $\beta$-induced ALK5 serine/threonine kinase activity, thus preventing the phosphorylation of Smad transcription factors and their subsequent gene activation $(35,36)$. The following results were observed: although SB525334 had no obvious effect on the expression of TGF- $\beta 1$, it inhibited the phosphorylation of Smad2 that was induced by TGF- $\beta 1$ and reversed the imbalance of MMPs/TIMPs. Furthermore, it decreased the expression of fibronectin, collagen type I and $\alpha$-SMA. These findings were observed in all corresponding PSC groups (over, control, Sh-1, Sh-2), which have significant differences compared with prior to using the TGF- $\beta$ inhibitor (Fig. 3C and D; Fig. 4). This could prove that TGF- $\beta 1 / \mathrm{Smad}$ pathway is essential for the fibrosis. As aforementioned we have clarified the relationship between galectin- 1 and TGF- $\beta 1 / \mathrm{Smad} 2$ expression. In addition, in the present study, upon applying the TGF- $\beta 1$ inhibitor, we observed that the subsequent effects were not dependent on whether galectin-1 expression was up- or downregulated. Thus, 
A

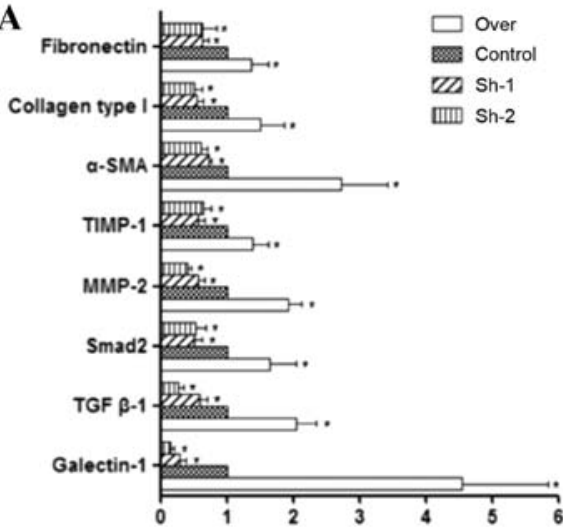

B

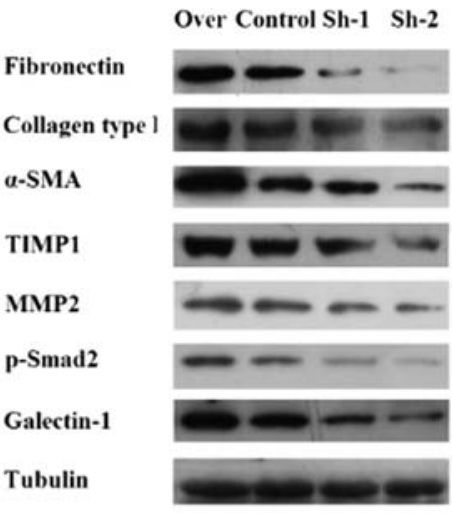

D

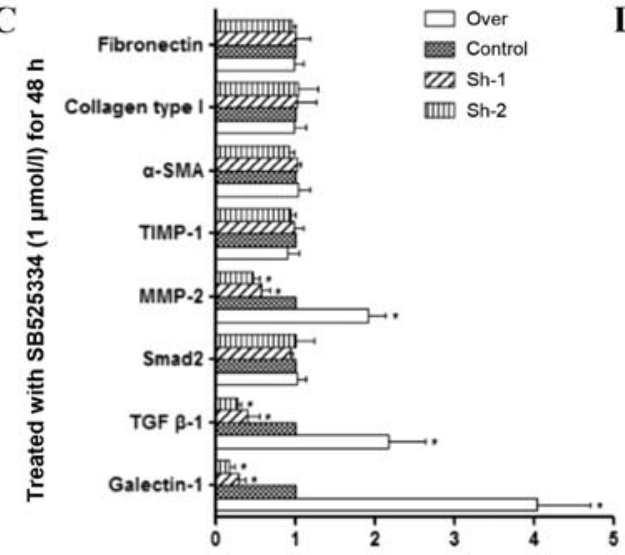

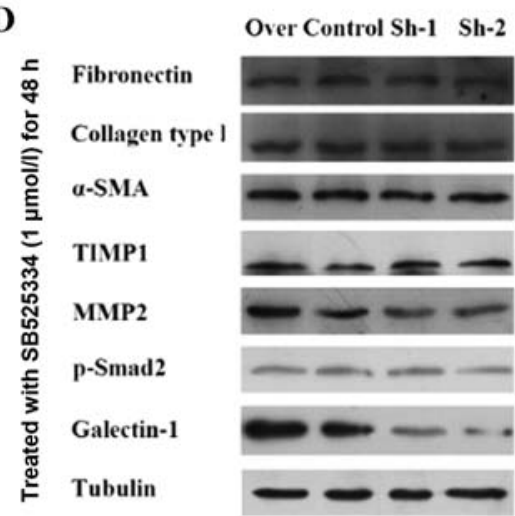

Figure 3. Fibrosis of activated PSCs via endogenous galectin-1-induced increase in TGF- $\beta 1 /$ Smad signaling and alteration of the MMP/TIMP ratio. (A and B) qRT-PCR and western blot analysis results showing the expression of fibrosis markers, TGF- $31 /$ Smad signaling factors, MMP-2 and TIMP-1 in PSCs with different levels of galectin-1 expression. (C and D) Changes in the expression of the same proteins after treatment with SB525334, a selective inhibitor of transforming growth factor- $\beta$ receptor I (ALK5, TGF- $\beta$ RI). The results of real-time PCR were calculated using the $2^{-\Delta \Delta C t}$ method. ${ }^{*} \mathrm{P}<0.05$, ${ }^{*} \mathrm{P}<0.01$, statistically significant differences compared with the control. Data are expressed as the means \pm SEM ( $n=3$ experiments/group).
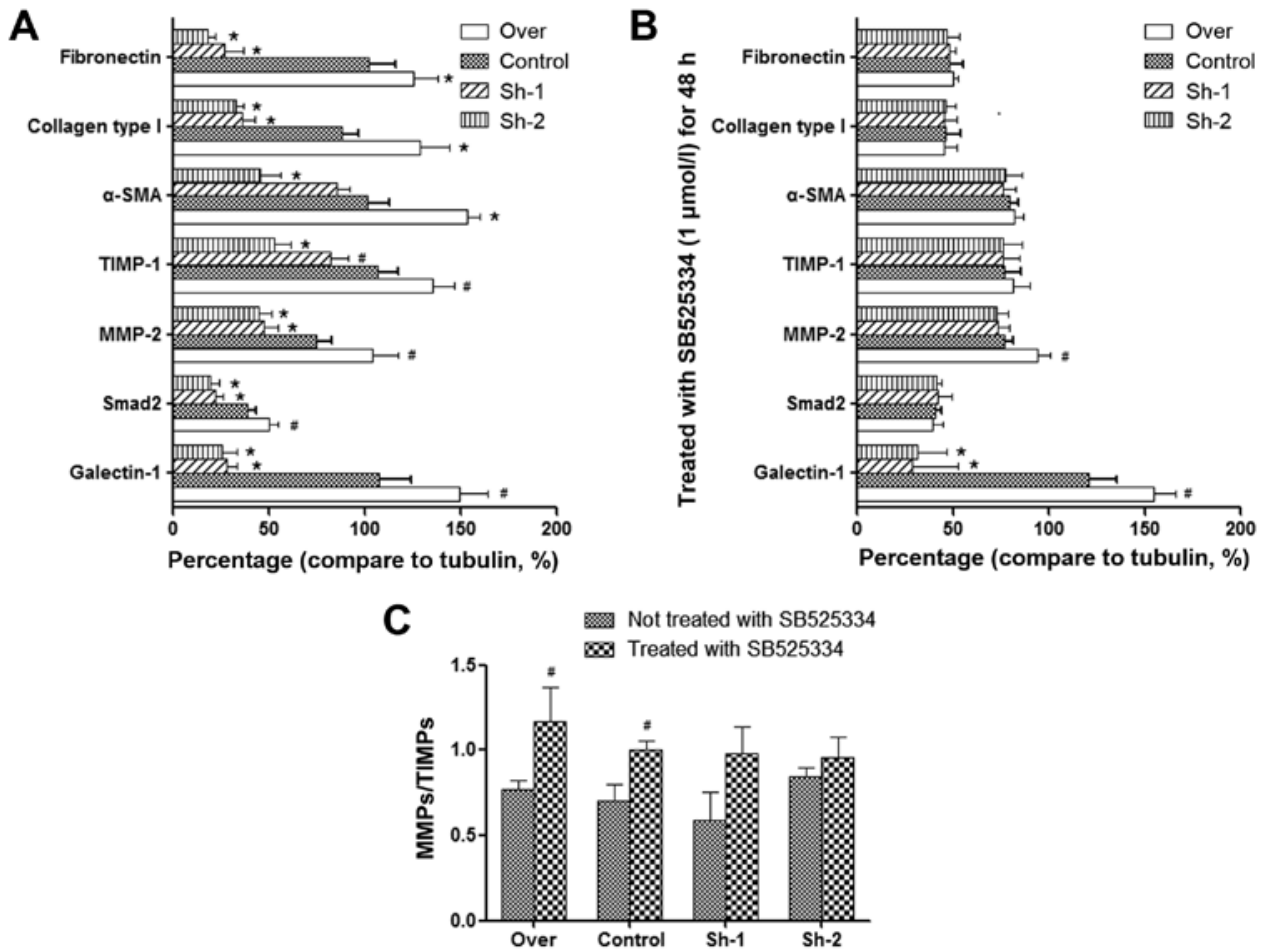

Figure 4. (A and B) Gray analysis of Fig. 3B and D (The ratio of the gray value of each protein band to the gray value of Tubulin). (C) The ratio of the MMP/TIMP gray value between the 'Not treated with SB525334' group and the 'Treated with SB525334' group. "P $<0.05$, "P<0.01, statistically significant differences compared with the control. 


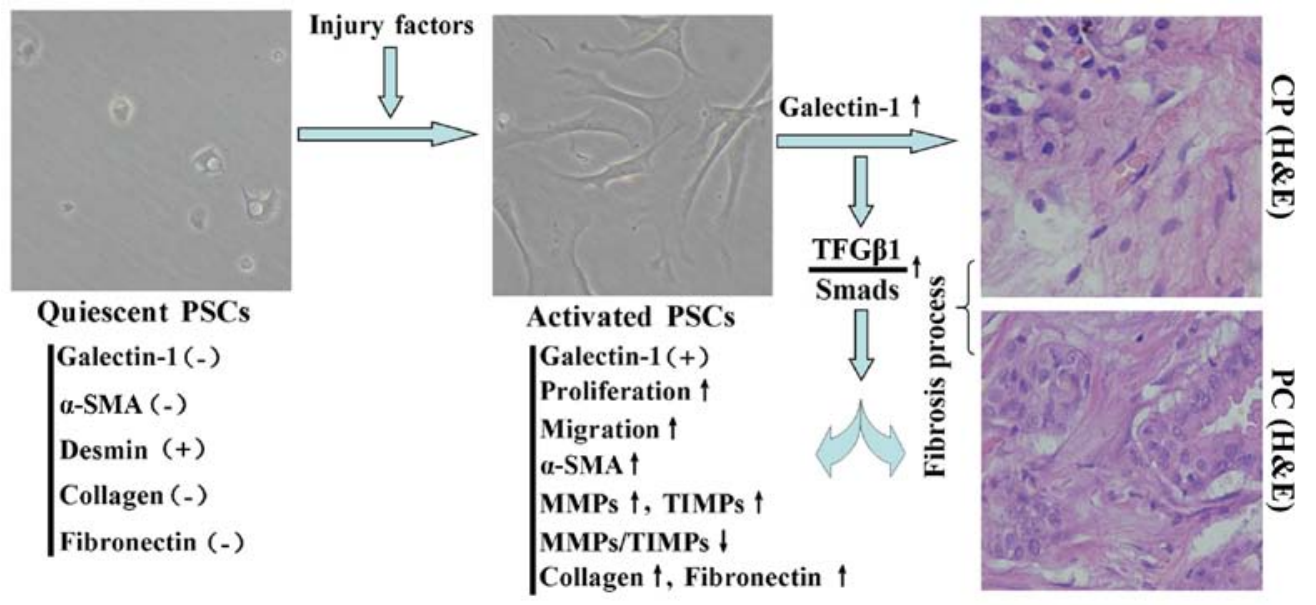

Figure 5. Flow chart depicting the mechanism by which endogenous galectin-1 expression in pancreatic stellate cells promotes fibrosis via the TGF- $\beta 1 /$ Smad pathway. By stimulating injury-related factors (such as ethanol metabolites and ROS), quiescent PSCs are activated and galectin-1 expression in PSCs is increased, which promotes fibrosis in the pancreas through the TGF- $\beta 1 /$ Smad signaling pathway-mediated imbalance of MMPs/TIMPs and a subsequent increase in the proliferation and migration of PSCs and expression of $\alpha$-SMA, fibronectin and collagen type I.

these results indicated that it was endogenous galectin-1 that induced TIMP expression by stimulating the TGF- $\beta 1 / \mathrm{Smad}$ signaling pathway, which decreased the degradation of ECM and increased the expression of fibronectin, collagen type I and $\alpha$-SMA, thus promoting the progression of PSC fibrosis.

\section{Discussion}

This study aimed to investigate the effect of PSC-derived galectin-1 on fibrogenesis in $\mathrm{CP} / \mathrm{PC}$ tissues and the underlying mechanisms. Fibrosis plays a vital role in the formation of tumor microenvironment and initiation of tumor angiogenesis (37), therefore the reversal of pancreatic fibrosis could be efficient for $\mathrm{CP} / \mathrm{PC}$ treatment (8). However, to date there are few therapeutic targets for the treatment of pancreatic fibrosis. We found that galectin-1 was expressed in CP/PC tissue and activated PSCs in vitro and that negative galectin-1 expression was observed in normal pancreatic tissue and PSCs in the quiescent state. Furthermore, we demonstrated that PSC-derived galectin-1 promoted the progression of fibrosis in PSCs via stimulation of the TGF- $\beta 1 /$ Smad signaling pathway. Collectively, these results indicated that PSC-derived galectin-1 may serve as a potential biomarker in therapeutic interventions for $\mathrm{CP} / \mathrm{PC}$.

Previous studies have revealed that endogenous galectin-1 expression is strongly induced upon activation of PSCs $(21,24,25)$. In accordance with these results, the immunohistochemical results of the present study also revealed strong galectin-1 staining in CP/PC tissues and activated PSCs. These findings indicated that PSCs play a role in pancreatic fibrosis in $\mathrm{CP} / \mathrm{PC}$ and that galectin-1 derived from PSCs may play a critical role in the progression of fibrosis in $\mathrm{CP} / \mathrm{PC}$. Furthermore, endogenous galectin- 1 was found to significantly increase the mRNA expression of collagen type I and fibronectin in PSCs. These results indicated that endogenous galectin- 1 induced the expression of soluble collagen and fibronectin by increasing their secretion or increasing the rate of their degradation. Thus, by altering the balance between ECM protein secretion and synthesis, endogenous galectin-1 may induce collagen and fibronectin synthesis in the ECM. Previous studies have revealed that collagen degradation promoted the regenerative response of hepatocytes during resolution of liver fibrosis (38). Thus, endogenous galectin-1 in PSCs may induce pancreatic degradation in patients with pancreatic injury by accelerating collagen synthesis.

Previous studies have revealed a 4.5 -fold increase in galectin-1 mRNA expression $(\mathrm{p}<0.01)$ in $\mathrm{CP} / \mathrm{PC}$ samples compared with normal controls, as well as upregulation of galectin-1 in fibroblasts. These findings indicated that galectin-1 plays a role in tissue remodeling in CP (39). In addition, PSCs exposed to exogenous galectin-1 proliferated at a higher rate and synthesized more collagen than the control cells (7). The present study also revealed that endogenous overexpression of galectin-1 in PSCs resulted in a significant increase in the proliferation and migration of PSCs. Furthermore, it resulted in an increase in the expression of TGF- $\beta 1$ [a known key profibrogenic factor (2)] and the phosphorylation of Smad2 and a consequent increase in the expression of fibronectin, collagen type I and $\alpha$-SMA. Contrasting results were observed in PSCs in which the expression of endogenous galectin-1 was silenced. Furthermore, the treatment of PSCs with SB525334, a selective inhibitor of TGF- $\beta$ receptor I, resulted in the inhibition of the phosphorylation of Smad2 (induced by TGF- $\beta 1$ ) and as a result, the expression of fibronectin, collagen type I and $\alpha$-SMA was also decreased. In accordance with these findings, it has been reported that galectin-1 may promote the TGF- $\beta 1$ induced differentiation of fibroblasts by sustaining nuclear localization of Smad2 and that knockdown of galectin-1 could decrease the phosphorylation and nuclear retention of Smad2, which may prevent the differentiation of fibroblasts (40). The TGF- $\beta /$ Smad signaling pathway has also been implicated in fibrosis development in a previous study (41). Collectively, all these results indicated that endogenous galectin-1 in PSCs significantly interacted with the TGF- $\beta 1 / \mathrm{Smad} 2$ pathway in a positive feedback loop, which may accelerate fibrosis of activated PSCs. Furthermore, TGF- $\beta 1$ may be a key promoter of ECM production and deposition of collagen type I and could trigger a Smad-dependent pathway to control 
galectin-1-induced pancreatic fibrosis in PSCs. Thus, TGF- $\beta 1$ and galectin-1 may work in synergy to tilt the balance towards fibrosis in $\mathrm{CP} / \mathrm{PC}$ (42). Therefore, galectin-1 expression in PSCs may present a potential therapeutic target for the antifibrosis treatment of $\mathrm{CP} / \mathrm{PC}$.

In the present study, we also examined MMP-2 and TIMP-1 synthesis by transformed cultured PSCs and their regulation by TGF- $\beta 1$. A significant role in the pathogenesis of PC and CP may be attributed to metalloproteinases (MMPs). The cellular basement membrane (BM) and ECM consist of collagen, laminin, elastin, fibronectin and proteoglycans which are subject to MMPs degradation (43). Degradation of the ECM is an essential step in tumor invasion and metastasis. Each MMP has different substrate specificities within the ECM and is important in the degradation of ECM. MMP activity is dependent on the levels of activated MMP and TIMPs (44). In the present study, our data revealed that galectin-1 induced an increase in MMP-2 secretion and an even greater increase in TIMP-1 expression in PSCs. Thus, galectin-1 facilitates the synthesis of ECM proteins by enhancing MMP activity, which has a pro-fibrogenic effect. There are several explanations for this effect. Firstly, MMP-2 is considered as an autocrine growth factor for stellate cells. Thus, increasing MMP-2 secretion may result in an increase in the number of PSCs and consequently induce collagen synthesis. Secondly, MMP-2 degrades normal basement membrane collagen (type IV); an increase in MMP-2 secretion may therefore, induce the deposition of pathological fibrillar collagen in the gland. However, TIMP-1 counteracts these effects and contributes to the development of pancreatic fibrosis. Therefore, when TIMP-1 expression is higher than MMP-2 expression, as observed in the activated PSCs in the present study, MMP activity is inhibited to some degree and the balance between ECM synthesis and degradation tilts towards fibrogenesis. These findings were similar to the above-mentioned studies and demonstrated that galectin-1 may play an important and hitherto unappreciated role in inducing pancreatic fibrosis (40).

This is the first study to investigate the effect of endogenous galectin-1 expression in PSCs in the fibrotic pancreatic tissue. By increasing the number of PSCs in the injured area and by promoting ECM synthesis, galectin-1 may act as a pro-fibrogenic protein in the process of injury of pancreatitis (Fig. 5). Further investigation into the molecular mechanisms and pathogenesis of pancreatic fibrosis using animal models would provide a better understanding of the process of fibrosis and clarify the adverse role of galectin-1 in pancreatic injury. This would be important in the development of novel approaches to antifibrotic therapy. Furthermore, the present study highlighted the role of galectin-1 in regulating the level of the fibrogenic cytokine marker TGF- $\beta 1$ and MMPs in the pathogenesis of $\mathrm{CP} / \mathrm{PC}$. This could mean that genotypes corresponding to high TGF- $\beta 1$ production may be associated with fibrogenesis in CP/PC $(45,46)$.

Based on the data presented in this study, we propose a mechanism for the induction of fibrosis by activated PSC-derived galectin-1 in CP/PC (Fig. 5): upon stimulation with a variety of pancreatic injury factors, quiescent PSCs were activated and their proliferation and migration were induced. Furthermore, the TGF- $\beta 1 /$ Smad signaling pathway was induced, which increased the expression of fibronectin, collagen type I and $\alpha$-SMA and altered the MMP/TIMP ratio. These events resulted in the reduction of ECM degradation and promoted fibrosis in $\mathrm{CP} / \mathrm{PC}$.

In conclusion, the findings of the present study indicated that PSCs and galectin-1 may have a potential for selective therapeutic treatments targeting fibrosis in $\mathrm{CP} / \mathrm{PC}$.

\section{Acknowledgements}

We thank Professor Lu Chun (Department of Microbiology and Immunology, Nanjing Medical University, Nanjing, China) for kindly providing the lentiviral packaging system consisting of pHAGE-CMVMCS-IZs Green, psPAX2 and pMD2.G. We would like to thank the native English speaking scientists of Elixigen Company (Huntington Beach, CA, USA) for editing the study. The present study was supported by grants from the National Natural Science Funding of China (no. 81572344), the Postdoctoral Science Foundation of China (no. 2013M530243), the Science and Technology Development Funding of Yangzhou City (no. 2012123), the Jiangsu Province Natural Science Foundation of China (no. BK20140495), the Six Big Talent Peak Projects of Jiangsu Province (no. 2014-WSW-078), the Postdoctoral Science Foundation of Jiangsu Province (2013), the training project of key talents of youth medicine in Jiangsu Province, (no. QNRC2016330), the 'Promote Health Development by Science and Technology' program of Jiangsu Province (no. KF201225) and the academic science and technology innovation fund for college students (x20160750, x20160753, x20160774 and x20160783).

\section{References}

1. Xue J, Sharma V, Hsieh MH, Chawla A, Murali R, Pandol SJ and Habtezion A: Alternatively activated macrophages promote pancreatic fibrosis in chronic pancreatitis. Nat Commun 6: 7158, 2015.

2. Staloch D, Gao X, Liu K, Xu M, Feng X, Aronson JF, Falzon M, Greeley GH, Rastellini C, Chao C, et al: Gremlin is a key profibrogenic factor in chronic pancreatitis. J Mol Med (Berl) 93: 1085-1093, 2015.

3. Tian L, Lu ZP, Cai BB, Zhao LT, Qian D, Xu QC, Wu PF, Zhu Y, Zhang JJ, Du Q, et al: Activation of pancreatic stellate cells involves an EMT-like process. Int J Oncol 48: 783-792, 2016.

4. Mahadevan D and Von Hoff DD: Tumor-stroma interactions in pancreatic ductal adenocarcinoma. Mol Cancer Ther 6: 1186-1197, 2007.

5. Hamada S, Masamune A, Yoshida N, Takikawa $T$ and Shimosegawa T: IL-6/STAT3 plays a regulatory role in the interaction between pancreatic stellate cells and cancer cells. Dig Dis Sci 61: 1561-1571, 2016.

6. Lin Z, Zheng LC, Zhang HJ, Tsang SW and Bian ZX: Antifibrotic effects of phenolic compounds on pancreatic stellate cells. BMC Complement Altern Med 15: 259, 2015.

7. Fitzner B, Walzel H, Sparmann G, Emmrich J, Liebe S and Jaster R: Galectin-1 is an inductor of pancreatic stellate cell activation. Cell Signal 17: 1240-1247, 2005.

8. Apte M, Pirola RC and Wilson JS: Pancreatic stellate cell: Physiologic role, role in fibrosis and cancer. Curr Opin Gastroenterol 31: 416-423, 2015.

9. Xiao W, Jiang W, Shen J, Yin G, Fan Y, Wu D, Qiu L, Yu G, Xing $\mathrm{M}, \mathrm{Hu} \mathrm{G}$, et al: Retinoic acid ameliorates pancreatic fibrosis and inhibits the activation of pancreatic stellate cells in mice with experimental chronic pancreatitis via suppressing the Wnt/ $\beta$-catenin signaling pathway. PLoS One 10: e0141462, 2015.

10. Incio J, Suboj P, Chin SM, Vardam-Kaur T, Liu H, Hato T, Babykutty S, Chen I, Deshpande V, Jain RK, et al: Metformin reduces desmoplasia in pancreatic cancer by reprogramming stellate cells and tumor-associated macrophages. PLoS One 10: e0141392, 2015. 
11. Takikawa T, Masamune A, Hamada S, Nakano E, Yoshida N and Shimosegawa T: miR-210 regulates the interaction between pancreatic cancer cells and stellate cells. Biochem Biophys Res Commun 437: 433-439, 2013

12. Tsang SW, Zhang HJ, Chen YG, Auyeung KK and Bian ZX: Eruberin A, a natural flavanol glycoside, exerts anti-fibrotic action on pancreatic stellate cells. Cell Physiol Biochem 36 2433-2446, 2015.

13. Nakamura T, Ito T, Uchida M, Hijioka M, Igarashi $H$, Oono $T$, Kato M, Nakamura K, Suzuki K, Jensen RT, et al: PSCs and GLP-1R: Occurrence in normal pancreas, acute/chronic pancreatitis and effect of their activation by a GLP-1R agonist. Lab Invest 94: 63-78, 2014.

14. He J, Sun X, Qian KQ, Liu X, Wang Z and Chen Y: Protection of cerulein-induced pancreatic fibrosis by pancreas-specific expression of Smad7. Biochim Biophys Acta 1792: 56-60, 2009.

15. Gao X, Cao Y, Yang W, Duan C, Aronson JF, Rastellini C Chao C, Hellmich MR and Ko TC: BMP2 inhibits TGF- $\beta$-induced pancreatic stellate cell activation and extracellular matrix formation. Am J Physiol Gastrointest Liver Physiol 304: G804-G813, 2013

16. Fitzner B, Brock P, Nechutova H, Glass A, Karopka T, Koczan D, Thiesen HJ, Sparmann G, Emmrich J, Liebe S, et al: Inhibitory effects of interferon-gamma on activation of rat pancreatic stellate cells are mediated by STAT1 and involve down-regulation of CTGF expression. Cell Signal 19: 782-790, 2007.

17. Böhm K, Teich N, Hoffmeister A, Mössner J, Keim V, Bödeker H and Gress TM: Transforming growth factor-beta-1 variants are not associated with chronic nonalcoholic pancreatitis Pancreatology 5: 75-80, 2005.

18. Liu J, Akanuma N, Liu C, Naji A, Halff GA, Washburn WK, Sun L and Wang P: TGF- $\beta 1$ promotes acinar to ductal metaplasia of human pancreatic acinar cells. Sci Rep 6: 30904, 2016

19. Tan R, Liu X, Wang J, Lu P, Han Z, Tao J, Yin C and Gu M Alternations of galectin levels after renal transplantation. Clin Biochem 47: 83-88, 2014.

20. Masamune A, Satoh M, Hirabayashi J, Kasai K, Satoh K and Shimosegawa T: Galectin-1 induces chemokine production and proliferation in pancreatic stellate cells. Am J Physiol Gastrointest Liver Physiol 290: G729-G736, 2006.

21. Tang D, Yuan Z, Xue X, Lu Z, Zhang Y, Wang H, Chen M, An Y, Wei J, Zhu Y, et al: High expression of Galectin-1 in pancreatic stellate cells plays a role in the development and maintenance of an immunosuppressive microenvironment in pancreatic cancer. Int J Cancer 130: 2337-2348, 2012.

22. Berberat PO, Friess H, Wang L, Zhu Z, Bley T, Frigeri L, Zimmermann A and Büchler MW: Comparative analysis of galectins in primary tumors and tumor metastasis in human pancreatic cancer. J Histochem Cytochem 49: 539-549, 2001

23. Mifková A, Kodet $O$, Szabo $P$, Kučera J, Dvořánková B, André S, Koripelly G, Gabius HJ, Lehn JM and Smetana K Jr: Synthetic polyamine BPA-C8 inhibits TGF- $\beta 1$-mediated conversion of human dermal fibroblast to myofibroblasts and establishment of galectin-1-rich extracellular matrix in vitro. ChemBioChem 15 $1465-1470,2014$.

24. Tang D, Zhang J, Yuan Z, Gao J, Wang S, Ye N, Li P, Gao S, Miao Y, Wang D, et al: Pancreatic satellite cells derived galectin-1 increase the progression and less survival of pancreatic ductal adenocarcinoma. PLoS One 9: e90476, 2014.

25. Tang D, Gao J, Wang S, Yuan Z, Ye N, Chong Y, Xu C, Jiang X, Li B, Yin W, et al: Apoptosis and anergy of $\mathrm{T}$ cell induced by pancreatic stellate cells-derived galectin-1 in pancreatic cancer. Tumour Biol 36: 5617-5626, 2015.

26. Tang D, Gao J, Wang S, Ye N, Chong Y, Huang Y, Wang J, Li B, Yin W and Wang D: Cancer-associated fibroblasts promote angiogenesis in gastric cancer through galectin-1 expression. Tumour Biol 37: 1889-1899, 2016.

27. Xue X, Lu Z, Tang D, Yao J, An Y, Wu J, Li Q, Gao W, Xu Z, Qian Z, et al: Galectin-1 secreted by activated stellate cells in pancreatic ductal adenocarcinoma stroma promotes proliferation and invasion of pancreatic cancer cells: An in vitro study on the microenvironment of pancreatic ductal adenocarcinoma. Pancreas 40: 832-839, 2011.

28. Apte MV, Haber PS, Applegate TL, Norton ID, McCaughan GW, Korsten MA, Pirola RC and Wilson JS: Periacinar stellate shaped cells in rat pancreas: Identification, isolation, and culture. Gut 43: 128-133, 1998
29. Ellenrieder V, Schneiderhan W, Bachem M and Adler G: Fibrogenesis in the pancreas. Rocz Akad Med Bialymst 49: 40-46, 2004

30. Jaskiewicz K,Nalecz A, Rzepko R and SledzinskiZ: Immunocytes and activated stellate cells in pancreatic fibrogenesis. Pancreas 26: 239-242, 2003.

31. Menke A and Adler G: TGFbeta-induced fibrogenesis of the pancreas. Int J Gastrointest Cancer 31: 41-46, 2002.

32. Li L, Bimmler D, Graf R, Zhou S, Sun Z, Chen J, Siech M and Bachem MG: PSP/reg inhibits cultured pancreatic stellate cell and regulates MMP/TIMP ratio. Eur J Clin Invest 41: 151-158, 2011.

33. Phillips PA, McCarroll JA, Park S, Wu MJ, Pirola R, Korsten M, Wilson JS and Apte MV: Rat pancreatic stellate cells secrete matrix metalloproteinases: Implications for extracellular matrix turnover. Gut 52: 275-282, 2003.

34. Li L, Bachem MG, Zhou S, Sun Z, Chen J, Siech M, Bimmler D and Graf R: Pancreatitis-associated protein inhibits human pancreatic stellate cell MMP-1 and -2, TIMP-1 and -2 secretion and RECK expression. Pancreatology 9: 99-110, 2009.

35. Laping NJ: Therapeutic uses of smad protein inhibitors: Selective inhibition of specific TGF-beta activities. IDrugs 2: 907-914, 1999.

36. Laping NJ, Everitt JI, Frazier KS, Burgert M, Portis MJ, Cadacio C, Gold LI and Walker CL: Tumor-specific efficacy of transforming growth factor-beta RI inhibition in Eker rats. Clin Cancer Res 13: 3087-3099, 2007.

37. Erkan M, Reiser-Erkan C, Michalski CW, Deucker S, Sauliunaite D, Streit S, Esposito I, Friess H and Kleeff J: Cancerstellate cell interactions perpetuate the hypoxia-fibrosis cycle in pancreatic ductal adenocarcinoma. Neoplasia 11: 497-508, 2009.

38. Suárez-Cuenca JA, Chagoya de Sánchez V, Aranda-Fraustro A, Sánchez-Sevilla L, Martínez-Pérez L and Hernández-Muñoz R: Partial hepatectomy-induced regeneration accelerates reversion of liver fibrosis involving participation of hepatic stellate cells. Exp Biol Med (Maywood) 233: 827-839, 2008.

39. Wang L, Friess H, Zhu Z, Frigeri L, Zimmermann A, Korc M, Berberat PO and Büchler MW: Galectin-1 and galectin-3 in chronic pancreatitis. Lab Invest 80: 1233-1241, 2000.

40. Lim MJ, Ahn J, Yi JY, Kim MH, Son AR, Lee SL, Lim DS, Kim SS, Kang MA, Han Y, et al: Induction of galectin-1 by TGF- $\beta 1$ accelerates fibrosis through enhancing nuclear retention of Smad2. Exp Cell Res 326: 125-135, 2014

41. Gu LZ, Sun H and Chen JH: Histone deacetylases 3 deletion restrains PM2.5-induced mice lung injury by regulating $N F-\kappa B$ and TGF- $\beta / \mathrm{Smad} 2 / 3$ signaling pathways. Biomed Pharmacother 85: 756-762, 2017.

42. Daroqui CM, Ilarregui JM, Rubinstein N, Salatino M Toscano MA, Vazquez P, Bakin A, Puricelli L, Bal de Kier Joffé E and Rabinovich GA: Regulation of galectin-1 expression by transforming growth factor beta 1 in metastatic mammary adenocarcinoma cells: Implications for tumor-immune escape. Cancer Immunol Immunother 56: 491-499, 2007.

43. Lekstan A, Olakowski M, Jabłońska B, Łabuzek K, Olakowska E, Filip I and Lampe P: Concentration of gelatinases and their tissue inhibitors in pancreatic inflammatory and neoplastic tumors and their influence on the early postoperative course. Pol Przegl Chir 85: 65-72, 2013.

44. Bramhall SR, Neoptolemos JP, Stamp GW and Lemoine NR: Imbalance of expression of matrix metalloproteinases (MMPs) and tissue inhibitors of the matrix metalloproteinases (TIMPs) in human pancreatic carcinoma. J Pathol 182: 347-355, 1997.

45. Bendicho MT, Guedes JC, Silva NN, Santana GO, dos Santos RR, Lyra AC, Lyra LG, Meyer R and Lemaire DC: Polymorphism of cytokine genes (TGF-beta1, IFN-gamma, IL-6, IL-10, and TNF-alpha) in patients with chronic pancreatitis. Pancreas 30 : 333-336, 2005.

46. Shek FW, Benyon RC, Walker FM, McCrudden PR, Pender SL, Williams EJ, Johnson PA, Johnson CD, Bateman AC, Fine DR, et al: Expression of transforming growth factor-beta 1 by pancreatic stellate cells and its implications for matrix secretion and turnover in chronic pancreatitis. Am J Pathol 160: 1787-1798, 2002. 